\title{
Diet and Chronic Urticaria: Dietary Modification as a Treatment Strategy
}

\author{
Joanna Jaros, ${ }^{1}$ Vivian Y. Shi, ${ }^{2}$ Rajani Katta ${ }^{3}$
}

1 University of Illinois College of Medicine, Chicago, IL, USA

2 Department of Medicine, Dermatology Division, University of Arizona at Tucson, AZ, USA

3 Dermatology, McGovern Medical School at UT Health, Bellaire, TX, USA

Key words: chronic urticaria, diet, pseudoallergens, food additives, histamine

Citation: Jaros J, Shi VY, Katta R. Diet and chronic urticaria: dietary modification as a treatment strategy. Dermatol Pract Concept. 2020;10(1):e2020004. DOI: https://doi.org/10.5826/dpc.1001a04

Accepted: September 25, 2019; Published: December 31, 2019

Copyright: (02019 Jaros et al. This is an open-access article distributed under the terms of the Creative Commons Attribution License, which permits unrestricted use, distribution, and reproduction in any medium, provided the original author and source are credited.

Funding: None.

Competing interests: The authors have no conflicts of interest to disclose.

Authorship: All authors have contributed significantly to this publication.

Corresponding author: Rajani Katta, MD, McGovern Medical School at UT Health, 6800 West Loop South, Ste 180, Bellaire, TX 77401. Email: info@kattamd.com

\begin{abstract}
Patients with chronic urticaria (CU) often ask about dietary modification. Research has indicated that specific dietary changes may be helpful in a subset of patients. Immunological food reactions are rare, but potential triggers of $\mathrm{CU}$ include those seen in certain settings, as in patients with a history of tick bites, a history of raw or marinated fish ingestion, or those with celiac disease. Nonimmunological food intolerances may also contribute, although mechanism of action is not well understood. Trials of pseudoallergen-free diets and low-histamine diets have resulted in partial remission in a subset of patients, while oral provocation testing has confirmed that some patients experience worsening of symptoms after ingestion of food additives, tomatoes, herbs, seafood, alcohol, and other foods. An increased prevalence of vitamin D deficiency has also been noted in patients with CU compared with healthy controls. While oral antihistamines remain the mainstay of therapy in $\mathrm{CU}$, education on potential dietary factors may be offered to a selection of the group of patients. For those at risk or reporting symptoms suggestive of celiac disease, vitamin D deficiency, delayed reactions to mammalian meat, or exposure to raw fish, further workup is recommended. While education on dietary modification may be offered to other patients, this approach may benefit only a subset, and no test is available to identify these patients. A minimum of 3 weeks may be needed to determine response, and only specific diets that have been systematically studied should be considered. Any elimination diet should be used with caution because of the potential for nutritional deficiencies.
\end{abstract}

\section{Introduction}

Patients with chronic urticaria (CU) often consider diet modification and discuss potential trigger foods. In this review, we evaluate the evidence behind potential dietary triggers for $\mathrm{CU}$ including immunological triggers (eg, galactose- $\alpha-1,3$-galactose [ $\alpha$-gal] in meat and Anisakis simplex in uncooked fish) and nonimmunological triggers. Nonimmunological triggers include compounds in tomatoes, food additives, herbs, wine, and other foods, as well as histamine in foods. The evidence 
and potential mechanisms behind these trigger foods is presented. Finally, we explore the increased prevalence of vitamin D deficiency in CU.

\section{Background}

Urticaria is a relapsing-remitting condition with significant impact on quality of life (QOL). Clinically, half of patients present with only cutaneous wheals, $10 \%$ with angioedema, and $40 \%$ with both [1] and individual lesions classically last $\leq 24$ hours. Current guidelines classify urticaria as acute vs chronic, based on duration less than or greater than 6 weeks. CU is further subclassified as spontaneous (no specific eliciting factor involved) vs inducible [2]. The lifetime prevalence in the general population is estimated at $9 \%$ and has significant impact on patients' QOL [3]. CU is particularly challenging for patients, as it is frequently treatment-resistant, long-standing, and idiopathic. This review focuses on dietary modifications for CU, with an emphasis on chronic spontaneous urticaria.

\section{Pathogenesis}

The etiology of CU is not well understood, and the majority of cases are considered idiopathic or due to autoreactivity [4]. At this time, neither European nor US guidelines recommend extensive laboratory testing or skin biopsy [2]. Therapy is focused on symptom management, with $\mathrm{H} 1$ antihistamines as the mainstay [5].

On a cellular level, the primary inflammatory cells are mast cells and basophils [3]. Activation of mast cells results in degranulation and release of preformed mediators, including vasoactive substances such as histamine and proinflammatory mediators such as tumor necrosis factor. Mast cells also release newly synthesized leukotrienes, prostaglandins, cytokines, and platelet activating factor [6]. These mediators in turn activate and recruit other immune cells [7]. This results in vasodilation, sensory nerve activation, and plasma extravasation, ultimately resulting in dermal edema and wheals [2]. Treatment with antihistamines significantly reduces these clinical findings [5].

A number of factors can cause mast cell activation, both immunological and nonimmunological. Immunoglobulin $\mathrm{E}$ (IgE)-mediated reactions are the primary immunological trigger [6]. Nonimmunological mechanisms are less well understood, but include medications, infections, food, and alcohol, among others [2].

Food allergies, which are due to defined immunological mechanisms, have been shown to be rare causes of CU. Sánchez et al administered food challenge tests (utilizing 410 common foods) to 164 patients with CU and 38 controls. Positive challenge tests occurred in only $1.2 \%$ of CU and $0.7 \%$ of control subjects [8]. Food IgE sensitization was similar in both groups $(17.5 \%$ vs $16.5 \%$, respectively) [8].

Therefore, despite the high frequency of self-reported food sensitivities, single foods are unlikely CU triggers. However, a number of other studies have presented intriguing results demonstrating that groups of foods may worsen CU. Specifically, elimination diets have improved symptoms in a subset of patients with $\mathrm{CU}$, while oral provocation testing (OPT) has resulted in flares.

\section{Chronic Urticaria and Diet}

Research has demonstrated that food allergies are extremely rare causes of CU. Food allergies that may result in symptoms of CU include a-gal allergy (meat) and A simplex allergy (fish nematode). Immunological reactions may occur due to gluten ingestion in celiac disease.

Food intolerances in $\mathrm{CU}$ are described more often than food allergies and are distinct from allergy [2]. These are defined as food-related symptoms that are objectively reproducible but that do not involve an immunological mechanism.

A number of foods have been reported to worsen CU symptoms. These "trigger" foods include alcohol, food additives, seafood, certain vegetables and fruits, fermented foods, and others [6]. Diagnostic and therapeutic strategies include food diaries, OPT, or elimination diets.

A review of 20 studies examined a total of 1,668 patients with $\mathrm{CU}$ who followed a systematic elimination diet approach. These consisted of pseudoallergen-free (PAF) diet, low-histamine (LH) diet, or fish avoidance diet used in conjunction with their antihistamine therapy [9]. Overall, these led to a combined complete remission in $4.9 \%$ and partial remission in $37.5 \%$ of subjects, with the highest rates reported for PAF and LH diets followed for a median duration of 3 weeks [9].

Partial remission rates in more than one-third of subjects are clearly worth further study, given the safety and low cost of these short-term dietary modifications. However, any study of CU must consider the significant spontaneous remission rate. Antia et al summarized the research on the natural history of CU [3] and noted variability, with one study reporting $32 \%$ of patients symptom-free after 3 years [10] and other studies reporting about half of patients experiencing remission after 1 year [11,12]. Lower remission rates are seen with more severe disease, while higher rates are seen with antihistamine treatment.

It is noteworthy that no control group was used in any of the aforementioned dietary studies, and many patients were allowed to use antihistamines. Therefore, it is difficult to compare these improvements with that expected via spontaneous remission. Despite these caveats, results were promising. The median duration of dietary change was only 3 weeks, and approximately onethird of patients reported improvement. Furthermore, several studies have doc- 
umented positive blinded OPT in a significant percentage of diet-responsive patients. Therefore, despite the lack of controlled trials, it appears that some patients have dietary triggers that may be contributing to CU.

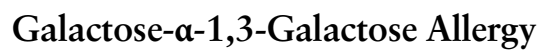

Galactose- $\alpha-1,3$-galactose ( $\alpha$-gal) is an oligosaccharide expressed on glycoproteins and glycolipids of nonprimate mammals [13,14]. It can be transmitted to humans by ticks, including the lone star tick Amblyomma americanum in the United States and other tick species in Europe and Australia [15]. Injection of a-gal during a tick bite results in development of IgE-mediated allergy to the a-gal antigen [16]. This antigen is then encountered when consuming mammalian meat (eg, beef, pork), as well as during infusions of the monoclonal antibody cetuximab [16]. In a study of 245 patients, symptoms included urticaria (93\%), gastrointestinal distress (64\%), and anaphylaxis (60\%) [17].

Symptom onset associated with a-gal hypersensitivity is typically delayed, occurring 2-6 hours after meat consumption [17]. Given the atypical timing, patients are frequently misdiagnosed with idiopathic CU [14]. In one study, 20 of 29 patients with a diagnosis of CU had detectable serum $\alpha$-gal antibodies, and of these, 95\% reported tick bites [18]. Avoidance of mammalian meat led to full remission in 9, and partial remission in 5 patients [18]. At this time, it is not known whether hypersensitivity will eventually improve.

In summary, a food diary may identify delayed responses to meat, with confirmation via blood test for $\alpha$-gal IgE antibodies. In those with antibodies, recommended treatment is allergen avoidance.

\section{Anisakis Simplex}

Hypersensitivity to A simplex, a sea fish nematode, may result in CU in coastal regions where uncooked fish is frequently consumed, with many of the published reports from Spain and Italy. The antigen may be present in raw, marinated, or smoked fish [19], and larvae may be implicated [20]. A randomized controlled trial of 213 Italian subjects with CU found that nearly $50 \%$ were sensitized to $A$ simplex on skin prick testing [21]. A 6-month diet excluding marinated and raw fish led to resolution of CU in $77 \%$, compared with $2 \%$ in controls [22]. Other studies have not been as promising. A smaller trial found no improvement in either sensitized or nonsensitized patients after 3 months of avoidance [22,23]. Additional studies have reported no change with fish-free diets, despite association between CU and $A$ simplex positivity on skin prick testing [24].

It is theorized that mast cell activation in patients with $\mathrm{CU}$ and parasitic infections, such as $A$ simplex, may involve the action of specific IgE, Th2 cytokine skewing, eosinophil recruitment to skin, and activation of the coagulation and complement systems [25]. With additional studies reporting benefit from fishfree diets [26], it is reasonable to either test at-risk patients or recommend trial avoidance. Research has also demonstrated that those allergic to $A$ simplex can consume frozen fish $\left(-20^{\circ} \mathrm{C}\right.$ for 48 hours) without risk of severe reaction [27]. Smoked fish is not recommended owing to potential reactivity to heat-stable $A$ simplex antigens [19].

\section{Gluten}

Autoimmune diseases, including celiac disease, have been linked to CU [28]. Case reports describe patients with celiac disease who develop CU following gluten ingestion $[29,30]$. Therefore, for patients reporting symptoms suggestive of celiac disease, further workup is recommended.

\section{Pseudoallergens}

Pseudoallergic reactions are not considered true allergy. Rather, they are a type of nonallergic hypersensitivity reaction. The mechanism of action is not well understood, as they do not act via IgE and are negative on skin testing. A wide number of compounds and foods may act as pseudoallergens [2]. These include natural compounds (including certain fruits, spices, vegetables), artificial additives (dyes, preservatives, flavorings), and vasoactive compounds (acetyl salicylic acid, histamine, and nitric oxide) [10]. Testing is typically with trial of elimination diet (ie, excluding compounds and foods reported as pseudoallergens) followed by OPT [31].

Despite the inherent difficulty, pseudoallergens have been studied in numerous trials. A systematic review summarized multiple PAF diet trials, encompassing 1,555 patients [9]. Overall, nearly $5 \%$ of patients showed complete remission, while $37 \%$ showed partial remission after median duration of 3 weeks. Although none had a control group, several trials reported OPT. Overall, observational studies of PAF diets have reported substantial reduction in medication usage [32], improved QOL scores [32], and significant decrease in leukotriene E4 levels [33].

Certain foods have been shown to trigger urticaria in diet-responsive patients during OPT, including tomatoes, food additives, wine, and herbs. In one study, $73 \%$ of patients reported improvement after only 3 weeks of PAF diet [34]. All responders later experienced symptoms after consuming a challenge meal rich in pseudoallergens [34]. OPT with distillation extract from whole tomatoes resulted in positive urticarial reactions in more than 50\% [34]. The culprit component remains unknown, as reactions were also common $(32 \%)$ in those given a tomato fraction lacking salicylic acid or p-hydroxybenzoate [34]. Of note, $46 \%$ of patients continuing dietary modification had full remission at 6 months [34]. In a similar study, 202 patients with CU underwent the same dietary modification for 3 months. Among 62\% of patients (126/202) who improved and later underwent OPT with placebo controls, $37 \%$ reacted to food additives [35]. 
In another study evaluating $33 \mathrm{CU}$ patients with known history of pseudoallergic reactions, $76 \%$ reacted to whole tomatoes, $50 \%$ to a panel of common food additives, $47 \%$ to herbal extracts in oil, and $44 \%$ to white wine [31]. Researchers then tested steam distillates and residues of these foods. Overall, patients reacted more strongly to tomato steam distillates $(45 \%)$ than residue $(15 \%)$ [31]. The tested residue was rich in histamine, salicylates, and proteins, and as only $15 \%$ of patients reacted to this residue, these are considered less likely triggers. When the tomato distillate was further analyzed by mass spectrometry, major ingredients included aldehydes, ketones, and alcohol, making these good targets for further study [31].

The exact mechanism of pseudoallergen activity is unknown, but one hypothesis centers on non-IgE dependent histamine release from mast cells. In vitro analysis of skin biopsy specimens found that tomato extract enhances histamine release from mast cells through co-stimulation of substance P or C5a, but not IgE [31]. Food components may also act via mast cell-independent effects. For example, it has been proposed that foods rich in cinnamaldehyde (eg, tomatoes and spices such as cinnamon and cloves) may worsen rosacea by activating transient receptor potential channels to promote neurogenic vasodilation [36,37].

Overall, PAF diets have improved symptoms in a subset of patients. However, efficacy across studies has been highly variable and no trial has been placebo-controlled. Regardless, many responders have demonstrated positive OPT. In addition, Zuberbier et al emphasize that pseudoallergic reactions are "known to be dose-dependent and frequently not restricted to just one agent" [31]. Therefore, single food challenge tests may not be as useful as elimination of multiple potential trigger foods.

In summary, while the value of PAF diet requires further investigation, espe- cially to identify potential responders and elucidate mechanism of action, it may be offered as adjunct therapy. Patients should be counseled that multiple foods should be excluded for up to 3 weeks before response may be seen. For responders, staged reintroduction of foods should then resume.

\section{Food Additives}

Food additives, including dyes, preservatives, and artificial sweeteners [38], have been studied as components of PAF diets, as well as independently. In one double-blind, placebo-controlled study of 838 patients, $31 \%$ experienced symptom improvement on a diet free of food additives [39]. One confounding factor is that such a diet may also be low in other pseudoallergens. Food additives seem unlikely to be isolated triggers, given results of a challenge trial. In this study, 100 patients with CU were challenged with 11 food additives, including dyes, preservatives, and artificial sweeteners [40]. Only 2 patients had an urticarial reaction on initial single-blind challenge, and neither reacted on second challenge. In conclusion, food additives alone do not appear to be a CU trigger, but a diet free of food additives (and presumably free of other potential triggers) may be helpful in a subset of patients.

\section{Histamine}

The vasoactive amine histamine is released from mast cells and is an important CU mediator, and antihistamines are considered as mainstay therapy [3]. Thus, researchers have evaluated LH diets as adjunct therapy for CU.

Ingestion of excess histamine may result in significant cutaneous as well as systemic symptoms, as in cases of histamine "poisoning" following ingestion of foods (such as certain fish) containing high levels of histamine. Affected individuals have experienced pruritus, angioedema, wheals, and systemic symptoms [41]. Histamine intolerance may occur as well, with symptoms fol- lowing ingestion of foods containing lesser levels of histamine. This is thought to arise from a mismatch between accumulated histamine (from single foods and combinations of foods) and an individual's capacity for histamine metabolism and clearance [42]. Some individuals may be more susceptible to ingested histamine, such as those with impaired diamine oxidase activity [43]. Intestinal diamine oxidase prevents dietary histamine uptake into the blood, and genetic factors, medical conditions, and alcohol may all reduce enzyme activity [44].

Certain foods contain higher levels of histamine, especially those with higher microbial activity or deterioration [44]. Seafood may contain higher levels depending on storage conditions and microbial exposure, as microbes convert amino acids such as histidine to biogenic amines [45]. Fermented foods are therefore also likely to have higher levels, including aged cheeses, dry sausage, and fermented soy [46]. Some plant foods have demonstrated higher levels as well, including tomatoes, eggplant, spinach, and avocado, although plant foods have demonstrated great variability in content [47].

Some CU patients report improvement on LH diets. In one study of 56 patients with both CU and gastrointestinal symptoms, $61 \%$ noted improvement after 3 weeks, with the greatest reduction in the strongly affected subgroup [48].

Another study also reported positive results, but indicated that response was unlikely due to decreased histamine ingestion [42]. In this study, 157 patients consumed a histamine-free, low pseudoallergen diet, with $46 \%$ reporting reduced CU activity [42]. All patients were then tested with oral histamine in a double-blind, placebo-controlled trial. While $17 \%$ reacted, no relationship was seen between response to diet and response to histamine. The researchers concluded that diets low in pseudoallergens may improve symptoms, but this response is not likely due to dietary his- 
tamine [42]. Further clouding the issue is that oral histamine challenge tests may provoke responses even in those with no history of food intolerance; in one study, close to $50 \%$ of volunteers reacted [49].

In conclusion, LH diets may be helpful in a subset of patients with CU, but the reason remains unclear. $\mathrm{LH}$ diets tend to be low in pseudoallergens [48], which is one possible explanation [42]. At this time, no accurate testing is available to determine potential responders.

\section{The Role of Vitamin D}

The potential immunomodulatory role of vitamin $\mathrm{D}$ in urticaria has received increasing attention [50]. A recent meta-analysis of 14 studies and 1,655 patients found that prevalence of vitamin D deficiency is significantly higher in patients with CU than in healthy controls [51]. Another meta-analysis confirmed these findings and proposed that CU is associated specifically with vitamin D deficiency, not insufficiency [52]. Vitamin D deficiency is defined by serum 25 -hydroxyvitamin $\mathrm{D}<20 \mathrm{ng} / \mathrm{dL}$ and insufficiency between 20 and 29 ng/dL [53].

Early interventional studies reported that vitamin D supplementation improved CU symptom severity, particularly in treatment-resistant patients with low baseline levels [54]. A later systematic review reported results of 7 studies describing CU improvement after highdose vitamin D supplementation, but doses and duration of use varied widely, indicating need for further study [55].

In summary, testing for vitamin D deficiency in those at risk, along with supplementation as appropriate, may be useful adjunctive therapy for patients with CU.

\section{Conclusions}

Immunological food allergies are extremely rare but might be potential causes of CU in certain settings, as in patients with a history of tick bites or a history of raw, marinated, or smoked fish

Table 1. Selected Tests for Suspected Dietary Factors in Chronic Urticaria

\begin{tabular}{|l|l|}
\hline $\begin{array}{l}\text { Patients reporting flares 2-6 hours following } \\
\text { ingestion of lamb, pork, or beef, especially in } \\
\text { those with history of tick bites }\end{array}$ & $\begin{array}{l}\text { IgE serum antibody test } \\
\text { for a-gal }\end{array}$ \\
\hline $\begin{array}{l}\text { History of raw, marinated, or smoked fish inges- } \\
\text { tion, particularly in coastal European regions }\end{array}$ & $\begin{array}{l}\text { IgE serum antibody test for } \\
\text { Anisakis simplex }\end{array}$ \\
\hline Symptoms suggestive of celiac disease & $\begin{array}{l}\text { Serum antibody testing for } \\
\text { celiac disease and referral to } \\
\text { gastroenterologist }\end{array}$ \\
\hline Risk factors for vitamin D deficiency & Serum vitamin D levels \\
\hline
\end{tabular}

$\alpha$-gal = galactose- $\alpha-1,3$-galactose $; \operatorname{IgE}=$ immunoglobulin $\mathrm{E}$. Table 2. Foods Reported as Nonimmunological
Chronic Urticaria Triggers

\begin{tabular}{|l|l|}
\multicolumn{1}{|c|}{ Food Group } & \multicolumn{1}{|c|}{ Reported Triggers (Suggest Avoidance) } \\
\hline Seafood & $\begin{array}{l}\text { - Avoid all except freshly caught and frozen fish, which is } \\
\text { then cooked }\end{array}$ \\
\hline Meat & - Aged sausages/smoked meats/processed meats \\
\hline Dairy & - Fermented dairy (aged cheeses, yogurt, sour cream) \\
\hline Vegetables & $\begin{array}{l}\text { - Tomatoes } \\
\text { - Spinach, eggplant, avocado } \\
\text { - Fermented vegetables such as sauerkraut and kimchi } \\
\text { - Overripe vegetables }\end{array}$ \\
\hline Fruits & $\begin{array}{l}\text { - All fruits } \\
\text { - All fruit juices }\end{array}$ \\
\hline Beverages & $\begin{array}{l}\text { - Alcohol } \\
\text { - Herbal tea }\end{array}$ \\
\hline Other & $\begin{array}{l}\text { - Any fermented foods } \\
\text { - Food additives (dyes, preservatives, artificial sweeteners) } \\
\text { - Spices and herbs } \\
\text { - Chocolate } \\
\text { - Chewing gum/candy }\end{array}$ \\
\hline
\end{tabular}

*Consider trial of 3-week avoidance as adjunct to antihistamine therapy in treatment-resistant patients.

*Includes foods listed on the pseudoallergen-free diet and the low-histamine diet.

ingestion. Nonimmunological reactions to foods are reported more frequently. Trials of specific elimination diets have reported high rates of response after an average duration of only 3 weeks. In addition, OPT has confirmed that some patients experience worsening of symptoms after ingestion of foods including food additives as well as natural foods such as tomatoes, herbs, seafood, alcohol, and others. The specific mechanism of action is not known.

In conclusion, while oral antihistamines are first-line therapy, educa- tion on potential dietary factors may be offered to a selected group of patients. A food diary is recommended, and those with suspected delayed meat or A simplex allergy should be offered testing. In those at risk or reporting symptoms suggestive of celiac disease or vitamin D deficiency, further workup is recommended (Table 1).

For other patients, a 3-week elimination diet trial may be considered, excluding foods known to be pseudoallergens or those high in histamine (Table 2). 
However, patients should be educated regarding several important caveats. This approach may benefit only a subset of patients, and at this time no test is commercially available to identify these patients. Only specific diets that have been systematically studied should be considered, and a minimum of 3 weeks is needed to determine response. Finally, any elimination diet has the potential to lead to nutritional deficiencies and should either be used only for short periods of time or with the aid of a nutritionist [2].

\section{References}

1. Powell RJ, Leech SC, Till S, Huber PAJ, Nasser SM, Clark AT. BSACI guideline for the management of chronic urticaria and angioedema. Clin Exp Allergy. 2015;45(3):547-565.

2. Zuberbier T, Aberer W, Asero R, et al. The EAACI/GA(2)LEN/ EDF/WAO guideline for the definition, classification, diagnosis and management of urticaria. Allergy. 2018;73(7):1393-1414.

3. Antia C, Baquerizo K, Korman A, Alikhan A, Bernstein JA. Urticaria: a comprehensive review: treatment of chronic urticaria, special populations, and disease outcomes. J Am Acad Dermatol. 2018;79(4):617-633.

4. Sheikhi A, Azarbeig M, Karimi H. Autohemotherapy in chronic urticaria: what could be the autoreactive factors and curative mechanisms? Ann Dermatol. 2014;26(4):526-527.

5. Sharma M, Bennett C, Carter B, Cohen SN. H1-antihistamines for chronic spontaneous urticaria: an abridged Cochrane Systematic Review. J Am Acad Dermatol. 2015;73(4):710-716.e4.

6. Theoharides TC, Valent P, Akin C. Mast cells, mastocytosis, and related disorders. N Engl J Med. 2015;373(2):163-172.

7. Gonzalez-de-Olano D, Alvarez-Twose I. Mast cells as key players in allergy and inflammation. J Investig Allergol Clin Immunol. 2018;28(6):365-378.

8. Sánchez J, Sánchez A, Cardona R. Dietary habits in patients with chronic spontaneous urticaria: evaluation of food as trigger of symptoms exacerbation. Dermatol Res Pract. 2018;2018:6703052.

9. Cornillier H, Giraudeau B, Samimi M, et al. Effect of diet in chronic spontaneous urticaria: a systematic review. Acta Derm Venereol. 2019;99(2):127-132.

10. Quaranta JH, Rohr AS, Rachelefsky GS, et al. The natural history and response to therapy of chronic urticaria and angioedema. Ann Allergy. 1989;62(5):421-424.

11. Kozel MM, Mekkes JR, Bossuyt PM, Bos JD. Natural course of physical and chronic urticaria and angioedema in 220 patients. $J$ Am Acad Dermatol. 2001;45(3):387-391.

12. Champion RH, Roberts SO, Carpenter RG, Roger JH. Urticaria and angio-oedema: a review of 554 patients. Br J Dermatol. 1969;81(8):588-597.

13. Commins SP, Click B, James H, Borish L, Platts-Mills TA. Delayed food allergy and anaphylaxis in patients with IgE to alpha-gal: a carbohydrate antigen that produces 'slowed' mast cell histamine release. Ann Allergy Asthma Immunol. 2009;103(5):A3.

14. Kennedy JL, Stallings AP, Platts-Mills TA, et al. Galactose-alpha-1,3-galactose and delayed anaphylaxis, angioedema, and urticaria in children. Pediatrics. 2013;131(5):e1545-e1552.

15. Steinke JW, Platts-Mills TA, Commins SP. The alpha-gal story: lessons learned from connecting the dots. J Allergy Clin Immunol. 2015;135(3):589-596; quiz 597.
16. Qian J, Liu T, Yang L, Daus A, Crowley R, Zhou Q. Structural characterization of $\mathrm{N}$-linked oligosaccharides on monoclonal antibody cetuximab by the combination of orthogonal matrix-assisted laser desorption/ionization hybrid quadrupole-quadrupole time-of-flight tandem mass spectrometry and sequential enzymatic digestion. Anal Biochem. 2007;364(1):8-18.

17. Wilson JM, Schuyler AJ, Workman L, et al. Investigation into the alpha-Gal syndrome: characteristics of 261 children and adults reporting red meat allergy. J Allergy Clin Immunol Pract. 2019;7(7):2348-2358.e4.

18. Pollack K, Zlotoff BJ, Borish LC, Commins SP, Platts-Mills TAE, Wilson JM. $\alpha$-Gal syndrome vs chronic urticaria. JAMA Dermatol. 2019;155(1):115-116.

19. Buquicchio R, Ventura MT, Traetta PL, Nenna S, Iadarola G, Magrone T. A multicenter study of IgE sensitization to Anisakis simplex and diet recommendations. Endocr Metab Immune Disord Drug Targets. 2018;18(2):170-174.

20. Sastre J, Lluch-Bernal M, Quirce S, et al. A double-blind, placebo-controlled oral challenge study with lyophilized larvae and antigen of the fish parasite, Anisakis simplex. Allergy. 2000;55(6):560-564.

21. Ventura MT, Napolitano S, Menga R, Cecere R, Asero R. Anisakis simplex hypersensitivity is associated with chronic urticaria in endemic areas. Int Arch Allergy Immunol. 2013;160(3):297-300.

22. Daschner A, De Frutos C, Valls A, et al. Preliminary data of the effect of a temporary diet without fish in a randomised clinical trial of Anisakis simplex sensitisation-associated chronic urticaria. Allergy. 2011;66:33.

23. López-Sáez MP, Zubeldia JM, Caloto M, et al. Is Anisakis simplex responsible for chronic urticaria? Allergy Asthma Proc. 2003;24(5):339-345.

24. Medina Fernández A, Barasona Villarejo M, Verdu Benhamu M, Moreno Aguilar C, Guerra Pasadas F. Anisakis allergy: clinical patterns and effectiveness of avoidance diet. Allergy. 2010;65:407.

25. Kolkhir P, Balakirski G, Merk HF, Olisova O, Maurer M. Chronic spontaneous urticaria and internal parasites-a systematic review. Allergy. 2016;71(3):308-322.

26. Gracia-Bara MT, Matheu V, Zubeldia JM, et al. Anisakis simplex-sensitized patients: should fish be excluded from their diet? Ann Allergy Asthma Immunol. 2001;86(6):679-685.

27. Trujillo MJ, Rodriguez A, Gracia Bara MT, et al. Dietary recommendations for patients allergic to Anisakis simplex. Allergol Immunopathol (Madr). 2002;30(6):311-314.

28. Kolkhir P, Borzova E, Grattan C, Asero R, Pogorelov D, Maurer M. Autoimmune comorbidity in chronic spontaneous urticaria: a systematic review. Autoimmun Rev. 2017;16(12):1196-1208.

29. Haussmann J, Sekar A. Chronic urticaria: a cutaneous manifestation of celiac disease. Can J Gastroenterol. 2006;20(4):291-293.

30. Peroni DG, Paiola G, Tenero L, et al. Chronic urticaria and celiac disease: A case report. Pediatr Dermatol. 2010;27(1):108-109.

31. Zuberbier T, Pfrommer C, Specht K, et al. Aromatic components of food as novel eliciting factors of pseudoallergic reactions in chronic urticaria. J Allergy Clin Immunol. 2002;109(2):343-348.

32. Magerl M, Pisarevskaja D, Scheufele R, Zuberbier T, Maurer M. Effects of a pseudoallergen-free diet on chronic spontaneous urticaria: a prospective trial. Allergy. 2010;65(1):78-83.

33. Akoglu G, Atakan N, Cakir B, Kalayci O, Hayran M. Effects of low pseudoallergen diet on urticarial activity and leukotriene levels in chronic urticaria. Arch Dermatol Res. 2012;304(4):257262. 
34. Zuberbier T, Chantraine-Hess S, Hartmann K, Czarnetzki BM. Pseudoallergen-free diet in the treatment of chronic urticaria: a prospective study. Acta Derm Venereol. 1995;75(6):484-487.

35. Pigatto PD, Valsecchi RH. Chronic urticaria: a mystery. Allergy. 2000;55(3):306-308.

36. Weiss E, Katta R. Diet and rosacea: the role of dietary change in the management of rosacea. Dermatol Pract Concept. 2017;7(4):31-37.

37. Pozsgai G, Bodkin JV, Graepel R, Bevan S, Andersson DA, Brain SD. Evidence for the pathophysiological relevance of TRPA1 receptors in the cardiovascular system in vivo. Cardiovasc Res. 2010;87(4):760-768.

38. Sahu SC. Food additives: a special issue of the journal Food and Chemical Toxicology. Food Chem Toxicol. 2017;107(Pt B):529.

39. Di Lorenzo G, Pacor ML, Mansueto P, et al. Food-additive-induced urticaria: a survey of 838 patients with recurrent chronic idiopathic urticaria. Int Arch Allergy Immunol. 2005;138(3):235-242.

40. Rajan JP, Simon RA, Bosso JV. Prevalence of sensitivity to food and drug additives in patients with chronic idiopathic urticaria. $J$ Allergy Clin Immunol Pract. 2014;2(2):168-171.

41. Colombo FM, Cattaneo P, Confalonieri E, Bernardi C. Histamine food poisonings: a systematic review and meta-analysis. Crit Rev Food Sci Nutr. 2018;58(7):1131-1151.

42. Siebenhaar F, Melde A, Magerl M, Zuberbier T, Church MK, Maurer M. Histamine intolerance in patients with chronic spontaneous urticaria. J Eur Acad Dermatol Venereol. 2016;30(10):1774-1777.

43. Reese I, Zuberbier T, Bunselmeyer B, et al. Diagnostic approach for suspected pseudoallergic reaction to food ingredients. J Dtsch Dermatol Ges. 2009;7(1):70-77.

44. Briguglio M, Dell'Osso B, Panzica G, et al. Dietary neurotransmitters: a narrative review on current knowledge. Nutrients. 2018;10(5). pii: E591.

45. Biji KB, Ravishankar CN, Venkateswarlu R, Mohan CO, Gopal TK. Biogenic amines in seafood: a review. J Food Sci Technol. 2016;53(5):2210-2218.
46. Stratton JE, Hutkins RW, Taylor SL. Biogenic amines in cheese and other fermented foods: a review. J Food Prot. 1991;54(6):460470.

47. Sanchez-Perez S, Comas-Baste O, Rabell-Gonzalez J, Veciana-Nogues MT, Latorre-Moratalla ML, Vidal-Carou MC. Biogenic amines in plant-origin foods: are they frequently underestimated in low-histamine diets? Foods. 2018;7(12).

48. Wagner N, Dirk D, Peveling-Oberhag A, et al. A popular mythlow-histamine diet improves chronic spontaneous urticaria-fact or fiction? J Eur Acad Dermatol Venereol. 2017;31(4):650-655.

49. Wohrl S, Hemmer W, Focke M, Rappersberger K, Jarisch R. Histamine intolerance-like symptoms in healthy volunteers after oral provocation with liquid histamine. Allergy Asthma Proc. 2004;25(5):305-311.

50. Straesser M, Palacios T, Kyin T, Borish L, Lawrence M. P55 seasonal correlations in chronic urticaria and allergic rhinitis. Ann Allergy Asthma Immunol. 2017;119(5):S39.

51. Tsai TY, Huang YC. Vitamin D deficiency in patients with chronic and acute urticaria: a systematic review and meta-analysis. $J \mathrm{Am}$ Acad Dermatol. 2018;79(3):573-575.

52. Wang X, Li X, Shen Y, Wang X. The association between serum vitamin D levels and urticaria: a meta-analysis of observational studies. G Ital Dermatol Venereol. 2018;153(3):389-395.

53. Holick MF. The vitamin D deficiency pandemic: approaches for diagnosis, treatment and prevention. Rev Endocr Metab Disord. 2017;18(2):153-165.

54. Boonpiyathad T, Pradubpongsa P, Sangasapaviriya A. Vitamin d supplements improve urticaria symptoms and quality of life in chronic spontaneous urticaria patients: a prospective case-control study. Dermatoendocrinol. 2014;6:e29727.

55. Tuchinda P, Kulthanan K, Chularojanamontri L, Arunkajohnsak S, Sriussadaporn S. Relationship between vitamin D and chronic spontaneous urticaria: a systematic review. Clin Transl Allergy. 2018;8:51. 